\title{
Treatment of severe nosocomial pneumonia: a prospective randomised comparison of intravenous ciprofloxacin with imipenem/cilastatin
}

Institut Clinic de Pneumologia i Cirurgia Toràcica, Servei de Pneumologia, UVIR, Universitat de Barcelona, IDIBAPS, Barcelona, Spain A Torres

Medizinische Klinik, Department of Pneumology, Allergology and Sleep Medicine,

Bergmannsheil

Klinikum der

Ruhr-Universität,

Bochum, Germany

T T Bauer

Servicio de Medicina Intensiva y Urgencias, Hospital, Universitario de Valme, Sevilla,

Spain

C León-Gil

Hospital Del Mar, Barcelona, Spain F Alvarez-Lerma

Hospital Virgen de la Arrixaca, Murcia, Spain

A Martínez-Pellús

Hospital Virgen del Rocío, Sevilla, Spain

F Castillo

S R Leal-Noval

Area de Vigilancia Intensiva, Hospital Clinic, Universitat de Barcelona, Barcelona, Spain

P Nadal

Servei Cures Intensives, Hospital Vall d'Hebrón, Barcelona, Spain M Palomar

Respiratory Intensive Care Unit, Hospital Clínico Universitario de Valencia, Spain

$\mathrm{J}$ Blanquer

Quimica Farmacéutica Bayer SA, Spain F Ros

Correspondence to: Dr A Torres atorres@medicina.ub.es

Received 5 January 2000 Returned to authors 3 March 2000

Revised version received 26 June 2000

Accepted for publication

8 August 2000

A Torres, T T Bauer, C León-Gil, F Castillo, F Alvarez-Lerma, A Martínez-Pellús, S R Leal-Noval, P Nadal, M Palomar, J Blanquer, F Ros

\section{Abstract}

Background-A prospective multicentre study was undertaken to compare the efficacy of intravenous ciprofloxacin or imipenem in the treatment of severe nosocomial pneumonia requiring mechanical ventilation.

Methods-Patients with a clinical suspicion of pneumonia were randomised to receive either ciprofloxacin $(800-1200 \mathrm{mg} /$ day) or imipenem (2-4 g/day) in doses adjusted for renal function and specimens of the lower respiratory tract were taken. Patients were included in the study when specimens showed significant growth for potentially pathogenic microorganisms in quantitative bacterial cultures $(n=75$, ciprofloxacin $41 / 75$ (55\%); imipenem $34 / 75$ $(45 \%))$. The clinical and bacteriological success rates were the primary and secondary efficacy variables. An intent-totreat analysis was performed for all randomised patients who received at least one dose of the study medication ( $n=149$, ciprofloxacin $72 / 149(48 \%)$, imipenem 77 I 149 (52\%)).

Results-The success rates were generally good, but neither the clinical success rates (ciprofloxacin, 29/41 (71\%), imipenem, $27 / 34(79 \%) ; 95 \%$ CI -10.8 to $28.1 ; p=$ 0.435) nor the bacteriological response rate (ciprofloxacin, 20/41 (49\%), imipenem, $17 / 34(50 \%)$; $95 \%$ CI -21.5 to 23.9 ; p $=1.0$ ) were significantly different between the study arms. Pseudomonas aeruginosa was recovered in $26 / 75$ patients (35\%) and clinical (ciprofloxacin, 10/14 (71\%), imipenem, 8/12 (67\%); 95\% CI -40.4 to $30.9 ; p=1.0)$ and bacteriological response rates (ciprofloxacin, $7 / 14(50 \%)$, imipenem, $3 / 12(25 \%), 95 \%$ CI -60.9 to $10.9, p=0.247$ ) were not significantly different in this subgroup of patients. Resistance of Pseudomonas aeruginosa developed in $5 / 26$ cases $(19 \%), 1 / 14(7 \%)$ to ciprofloxacin and $4 / 12(33 \%)$ to imipenem $(p=0.147)$, and the mortality was $12 / 75$ $(16 \%)$ with no difference between treatment groups (ciprofloxacin, 8/41(24\%), imipenem $4 / 34(17 \%) ; p=0.362)$. The clinical response was evaluable in 109/149 patients $(73 \%)$ in the intent-to-treat analysis and was successful in 74/109 patients $(68 \%)$. The clinical response rates were also not significantly different in the intent-to-treat analysis (ciprofloxacin,
34/52 (65\%), imipenem, $40 / 57$ (70\%); 95\% CI -12.8 to $22.3 ; p=0.746)$.

Conclusions-Treatment with either ciprofloxacin or imipenem was effective in a selected group of patients with microbiologically confirmed, severe nosocomial pneumonia requiring mechanical ventilation. Although no differences between the study medication could be documented in this trial, smaller differences between treatment arms may have been missed because of sample size limitations.

(Thorax 2000;55:1033-1039)

Keywords: pneumonia; treatment; ciprofloxacin; imipenem

Nosocomial pneumonia is associated with a high mortality despite the availability of broad spectrum antibiotics. ${ }^{1}$ The first appropriate treatment measure is the parenteral administration of an empirical antibiotic. ${ }^{2}$ Broad spectrum antibiotic coverage is generally advisable because Gram negative bacteria often cause severe bacterial nosocomial pneumonia and a multibacterial aetiology is frequent. The efficacy of single antibiotic treatment in nosocomial pneumonia is controversial and depends largely on the type of causative microorganisms. Epidemiological approaches have led to the establishment of distinct patient groups with presumed causative agents according to the presence of risk factors, time of onset, and severity of the episode. ${ }^{1}$ However, few data are available on the treatment of nosocomial pneumonia with a single drug, regardless of the causative agent.

Ciprofloxacin and imipenem are two broad spectrum antimicrobial agents of different chemical class that have been widely investigated separately for the treatment of lower respiratory tract infections. Some trials have described only initial experiences with either drug $^{3-10}$ while others have used a randomised design for the comparison of either ciprofloxacin or imipenem with another antibiotic. ${ }^{11-21}$ The efficacy of ciprofloxacin and imipenem has been compared directly for the treatment of severe clinical infection, ${ }^{22}$ pneumonia in ventilated patients, ${ }^{23}$ and in one randomised comparison for the treatment of severe pneumonia. ${ }^{24}$ Treatment with ciprofloxacin was associated with a significantly higher clinical response rate, especially when Pseudomonas aeruginosa or Enterobacteriaceae were recovered from the baseline bacterial cul- 
ture. ${ }^{24}$ However, this trial also included patients with community acquired pneumonia, with and without ventilatory support, and, more importantly, did not use quantitative bacterial cultures of lower respiratory tract specimens to confirm pneumonia.

We have therefore compared the efficacy of ciprofloxacin with that of imipenem in a well defined population of patients with microbiologically confirmed, severe nosocomial pneumonia requiring mechanical ventilation.

\section{Methods}

PATIENT SELECTION

This study was an open label, prospective, randomised trial for the comparison of efficacy and safety of intravenous ciprofloxacin with that of intravenous imipenem/cilastatin for the treatment of patients with severe nosocomial pneumonia. All adults (aged $>18$ years) with clinical suspicion of nosocomial pneumonia in nine clinical centres in Spain were eligible for entry into the study. Patients were enrolled during one year and statistical analysis was carried out at one trial centre (AT). The study was approved by the ethical committee and conducted in accordance with its guidelines. The following clinical definitions were applied:

Clinical suspicion of pneumonia: new and persistent infiltrates on the chest radiograph attributable to pulmonary infection and at least two of the following criteria: (1) fever $\left(\geqslant 38.3^{\circ} \mathrm{C}\right)$; (2) leukopenia or leukocytosis (WBC $\leqslant 4000$ or $\geqslant 12000 / \mathrm{mm}^{3}$, or (3) purulent tracheal secretions.

Nosocomial pneumonia: clinical suspicion of pneumonia present $\geqslant 72$ hours after hospital admission.

Severe nosocomial pneumonia: patients required mechanical ventilation with $\geqslant 40 \% \mathrm{FIO}_{2}$ to maintain a $\mathrm{PaO}_{2}$ of $\geqslant 8 \mathrm{kPa}(60 \mathrm{~mm} \mathrm{Hg})$.

Microbiologically confirmed nosocomial pneumonia: potentially pathogenic microorganism (PPM) recovered from the lower respiratory tract showed significant growth in quantitative bacterial cultures or if blood cultures or pleural effusions showed bacterial growth (see section on bacteriological procedures for definitions).

All patients with a clinical suspicion of pneumonia were randomised after written informed consent was obtained from the next of kin. Closed envelopes were distributed to each centre before the study to ensure randomisation. Patients were included in the study if the study medication had been given for $\geqslant 3$ days, if pneumonia could be confirmed microbiologically (see definition above), and if the isolated microorganism was sensitive to the study medication (see section on bacteriological procedures for definition).

The following exclusion criteria were applied before randomisation: allergy to a fluoroquinolone, $\beta$-lactam or penicillin; patient pregnant or lactating; changes in systemic antibiotic therapy during the five days before enrolment, significant leukopenia $\left(\leqslant 1000 / \mathrm{mm}^{3}\right)$, underlying immunocompromising disease or immuno- suppressive therapy, and application of study medication 30 days before enrolment to the study.

\section{BACTERIOLOGICAL PROCEDURES}

Microorganisms were grouped as potentially pathogenic microorganisms (PPMs) and nonPPMs. The following microorganisms were regarded as non-PPMs: Streptococcus spp except Streptococcus pneumoniae, coagulase negative staphylococci, Neisseria spp, and Candida spp. Polymicrobial growth was present with two or more PPMs in culture. Lower respiratory tract specimens (tracheobronchial aspirate (TBAS), bronchoalveolar lavage (BAL) fluid, and protected specimen brush (PSB)) were cultured quantitatively for the microbiological confirmation of suspected pneumonia. The growth of PPMs was regarded significant when the following thresholds were exceeded: TBAS $\geqslant 10^{5}$ colony forming units (cfu) $/ \mathrm{ml}$, PSB $\geqslant 10^{3} \mathrm{cfu} / \mathrm{ml}$, and BAL fluid $\geqslant 10^{4} \mathrm{cfu} / \mathrm{ml}^{25}{ }^{26}$ Any growth of a PPM in blood or pleural effusion was regarded significant.

Susceptibility testing of bacterial isolates was performed according to standard methods. ${ }^{27}$ According to the minimal inhibitory concentrations (MIC) the susceptibility was rated

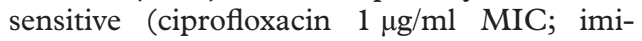
penem $4 \mu \mathrm{g} / \mathrm{ml} \mathrm{MIC}$ ), intermediate (ciprofloxacin $2 \mu \mathrm{g} / \mathrm{ml}$ MIC; imipenem $8 \mu \mathrm{g} / \mathrm{ml}$ MIC), and resistant (ciprofloxacin $4 \mu \mathrm{g} / \mathrm{ml}$ MIC; imipenem $16 \mu \mathrm{g} / \mathrm{ml}$ MIC). Development of resistance was assumed when the bacteria were susceptible or moderately susceptible to the study drug in baseline culture and a strain resistant to the study drug was isolated in one follow up culture.

TREATMENT

The study medication dose was adjusted for all patients according to renal creatinine clearance. The unadjusted doses permitted in this trial were $800-1200 \mathrm{mg}$ per day for ciprofloxacin and 2-4 g/day for imipenem. No other antimicrobial agent was allowed during the study except those initiated more than five days before the study.

\section{EFFICACY PARAMETERS}

The clinical response was determined by assessing signs and symptoms of respiratory infection, as well as comparing basal chest radiographs with those obtained at the end of the study. The clinical response was rated as (1) a cure if signs and symptoms related to pulmonary infection had disappeared, (2) failure if lessening of symptoms related to pulmonary infection was rated insufficient or additional treatment was necessary, (3) improved if neither cure nor failure were applicable, and (4) indeterminate if a clinical evaluation was not possible.

The bacteriological response was classified as follows: (1) eradication (elimination of the initial causative microorganism documented in at least one LRTS during the study), (2) presumed eradication (no follow up specimen available and clinical response improved or cure), (3) persistence (continuous isolation of 
the basal causative organism), (4) superinfection (elimination of the basal causative agent but isolation of another PPM during follow up), and (5) presumed failure (no follow up specimen available and clinical response failure). Treatment success was assumed if eradication or presumed eradication were present. Treatment failure was rated as persistence, superinfection, or presumed failure.

All adverse clinical events or laboratory abnormalities occurring during treatment or within seven days of discontinuation of the study drug were reported and evaluated for possible association with the study drug.

\section{STATISTICAL METHODS}

The primary objective of this study was to compare the clinical response between the two study arms in the predefined study population. An intent-to-treat analysis was performed to disclose possible bias induced by our stringent selection criteria. Indeterminate clinical responses were not included in the calculation of resolution rates. The secondary objective of this study was the comparison of the bacteriological response between the study arms. Moreover, data on safety of the study drugs and mortality within the treatment period were reported.

Data are reported as counts or mean (SD) values. Frequencies were compared using the $\chi^{2}$ test or Fisher's exact test (expected cell frequency <5) and mean values by the MannWhitney $\mathrm{U}$ test. Comparisons of success rates

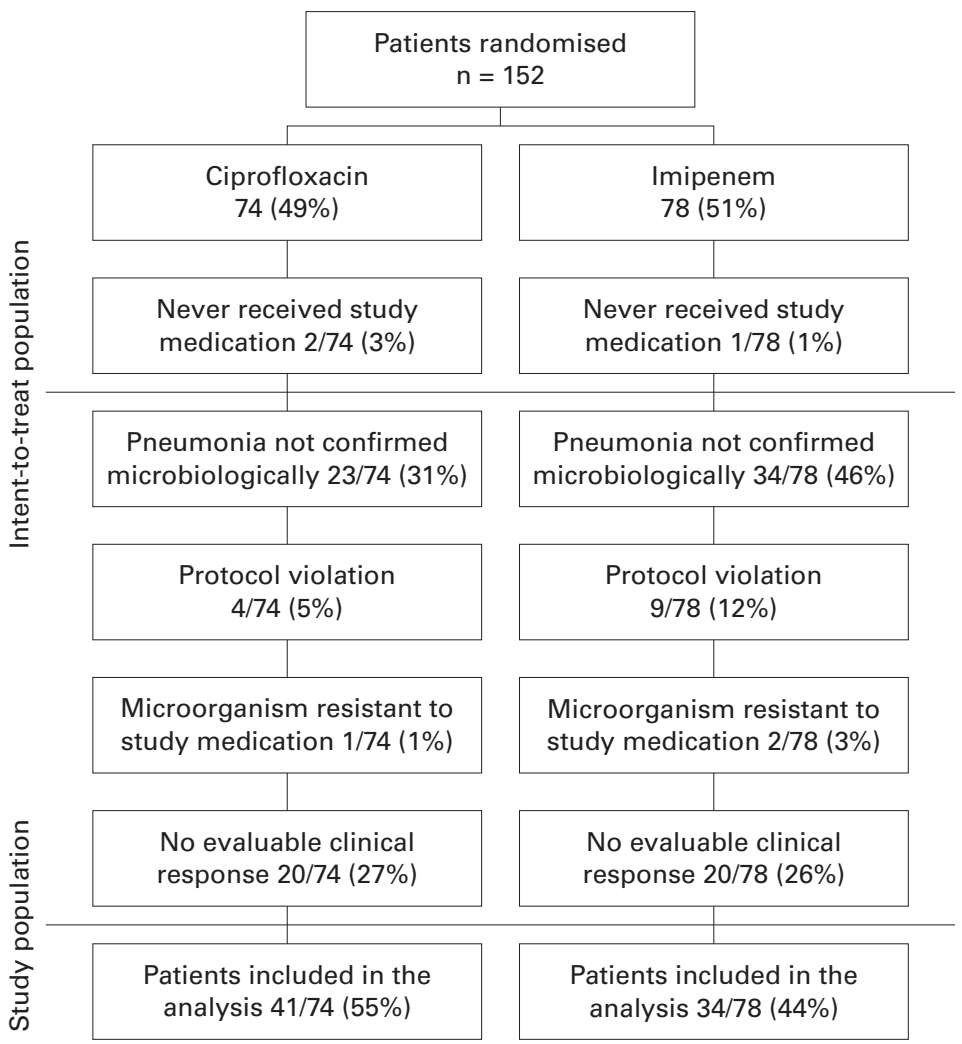

Figure 1 Trial profile. Only patients with microbiologically confirmed pneumonia were included in the study population $(n=75)$. The intent-to-treat analysis was performed on all patients who had been randomised and received at least one dose of the study medication $(n=149)$. The total number of patients excluded in smaller than the sum of individual causes because there were multiple causes in some patients. between treatment arms and for recovered organisms are reported with $95 \%$ confidence intervals for comparison of proportions. All data were processed using the SPSS version 9.0 on a Windows 95 operating system.

\section{Results}

STUDY POPULATION

One hundred and fifty two patients were randomised and three patients never received the study medication (fig 1). The clinical suspicion of pneumonia could not be confirmed microbiologically in 57 of 152 patients $(38 \%)$ and protocol violation made the exclusion of 13 patients $(9 \%)$ necessary (one had no need for mechanical ventilation, two had been in hospital $<72$ hours before ICU admission, three had received concomitant antimicrobial treatment before the study, and in seven cases a pretreatment bacterial culture was not available). The microorganisms found in baseline cultures were resistant to the study medication in three cases $(2 \%)$ and in $14(9 \%)$ the clinical response was not evaluable (fig 1). The final study population therefore comprised 75/152 patients $(49 \%), 41$ of whom $(55 \%)$ had been randomised to ciprofloxacin and $34(45 \%)$ to imipenem.

To correct for a possible bias induced by the stringent entry criteria an intent-to-treat analysis was performed on all randomised patients who had received at least one dose of the study medication ( $\mathrm{n}=149$ (98\%), 72 (48\%) receiving ciprofloxacin and $77(52 \%)$ receiving imipenem).

The clinical characteristics between the two study arms for both populations are shown in table 1 . No significant differences were found in either the study population or in the population included in the intent-to-treat analysis. Severity of respiratory failure as measured by the alveolar-arterial oxygen tension gradient $\left(\mathrm{P}(\mathrm{A}-\mathrm{a}) \mathrm{O}_{2}\right)$ and the fractional inspired oxygen ratio $\left(\mathrm{FIO}_{2}\right)$ and severity of the illness as measured by the APACHE II score were not significantly different.

\section{ANALYSIS OF STUDY POPULATION}

Study medication

The mean duration of treatment was not significantly different between the ciprofloxacin and imipenem groups (9.3 (3.8) versus 10.1 (3.2) days; $p=0.372)$. The mean daily dose was 1035 (163) mg/day (14.5 (3.4) mg/ $\mathrm{kg} /$ day) for ciprofloxacin and 2655 (674) $\mathrm{mg} /$ day $(38.4(10.6) \mathrm{mg} / \mathrm{kg} /$ day) for imipenem.

\section{Bacteriological data}

The microorganisms recovered in the baseline culture are shown in table 2. The most commonly recovered causative organisms in the study population were $P$ aeruginosa ( $\mathrm{n}=$ 26), Haemophilus influenzae ( $\mathrm{n}=21)$, and $S$ pneumoniae $(\mathrm{n}=15)$. All microorganisms were sensitive to the study medication (see definition of study population). Although there was a trend towards a higher frequency of $H$ influen$z a e$ isolates in the imipenem group, no statistically significant differences were detected. The proportion of cultures with polymicrobial 
Table 1 Demographic characteristics of the study population and the intent-to-treat population

\begin{tabular}{|c|c|c|c|c|}
\hline & \multicolumn{2}{|c|}{ Study population $(n=75)$} & \multicolumn{2}{|c|}{ Intent-to-treat population $(n=149)$} \\
\hline & Ciprofloxacin $(n=41)$ & Imipenem $(n=34)$ & Ciprofloxacin $(n=72)$ & Imipenem $(n=77)$ \\
\hline Men, n (\%) & $31(75.6 \%)$ & $25(73.5 \%)$ & $53(73.6 \%)$ & $57(74.0 \%)$ \\
\hline Women, n (\%) & $10(24.4 \%)$ & $9(26.5 \%)$ & $19(26.4 \%)$ & $20(26.0 \%)$ \\
\hline Mean (SD) age (years) & $64(14)$ & $61(17)$ & $60(16)$ & $62(16)$ \\
\hline Mean (SD) weight (kg) & $74(13)$ & $71(10)$ & $72(12)$ & $71(11)$ \\
\hline Mean (SD) BMI $\left(\mathrm{kg} / \mathrm{m}^{2}\right)$ & $26.7(4.6)$ & $25.9(3.5)$ & $26.1(4.0)$ & $26.0(3.8)$ \\
\hline No with one or more comorbid illness & $41(100 \%)$ & $30(88 \%)$ & $70(97 \%)$ & $71(92 \%)$ \\
\hline \multicolumn{5}{|l|}{ Type of comorbid illness ${ }^{\star}, \mathrm{n}(\%)$} \\
\hline COPD & $16(39 \%)$ & $12(35 \%)$ & $20(28 \%)$ & $29(38 \%)$ \\
\hline Other respiratory illness & $3(7 \%)$ & $1(4 \%)$ & $2(3 \%)$ & $2(3 \%)$ \\
\hline Cardiovascular/vascular & $14(34 \%)$ & $16(47 \%)$ & $28(39 \%)$ & $33(43 \%)$ \\
\hline Neurological & $10(24 \%)$ & $10(29 \%)$ & $17(24 \%)$ & $23(30 \%)$ \\
\hline Diabetes mellitus & $6(15 \%)$ & $6(18 \%)$ & $9(13 \%)$ & $7(9 \%)$ \\
\hline Gastrointestinal & $9(2 \%)$ & $3(9 \%)$ & $10(14 \%)$ & $4(5 \%)$ \\
\hline Neoplasm & $7(17 \%)$ & 0 & $12(17 \%)$ & $4(5 \%)$ \\
\hline Renal & $2(5 \%)$ & 0 & $2(3 \%)$ & $4(5 \%)$ \\
\hline Other & $3(7 \%)$ & 0 & $3(4 \%)$ & $1(1 \%)$ \\
\hline Mean (SD) $\mathrm{P}(\mathrm{A}-\mathrm{a}) \mathrm{O}_{2}$ & $218(113)$ & $215(154)$ & $257(157)$ & $213(144)$ \\
\hline Mean (SD) $\mathrm{PaO}_{2} / \mathrm{FIO}_{2}$ ratio & $206(93)$ & $223(86)$ & $204(101)$ & $229(124)$ \\
\hline Mean (SD) APACHE score & $13.8(7.5)$ & $13.9(8.6)$ & $13.9(7.7)$ & $14.7(7.6)$ \\
\hline Mean (SD) leucocyte count $/ \mathrm{mm}^{3} \dagger$ & $14.5(5.9)$ & $12.6(5.5)$ & $14.5(5.6)$ & $12.6(4.9)$ \\
\hline Mean (SD) creatinine $(\mathrm{mg} / \mathrm{dl})$ & $1.3(1.2)$ & $0.9(0.4)$ & $1.2(1.1)$ & $1.0(0.6)$ \\
\hline Mean (SD) body temperature $\left({ }^{\circ} \mathrm{C}\right)$ & $37.8(0.9)$ & $37.9(1.1)$ & $37.8(1.0)$ & $37.8(0.9)$ \\
\hline Mean (SD) PEEP $\left(\mathrm{cm} \mathrm{H}_{2} \mathrm{O}\right)$ & $5.7(2.8)$ & $5.7(3.3)$ & $6.2(3.1)$ & $6.0(3.4)$ \\
\hline (no $(\%)$ patients with PEEP) & $(15(36.6 \%))$ & $(17(45.9 \%))$ & $(34(47.2 \%))$ & $(29(37.6 \%))$ \\
\hline No $(\%)$ patients with PEEP $\geqslant 5 \mathrm{~cm} \mathrm{H}_{2} \mathrm{O}$ & $22(53.7 \%)$ & $18(52.9)$ & $38(52.8 \%)$ & $36(46.8)$ \\
\hline
\end{tabular}

$\mathrm{BMI}=$ body mass index $\mathrm{P}(\mathrm{A}-\mathrm{a}) \mathrm{O}_{2}=$ alveolar-arterial oxygen tension gradient; $\mathrm{PaO}_{2}=$ arterial oxygen tension; $\mathrm{FIO}_{2}=$ fractional inspired oxygen concentration; $\mathrm{PEEP}$ $=$ positive end expiratory pressure required on enrollment to the study.

$\star$ Sum of single percentages higher than $100 \%$ due to multiple comorbid illnesses.

†Mann-Whitney U test, $\mathrm{p}=0.165$.

Table 2 Microorganisms recovered in the study population

\begin{tabular}{|c|c|c|c|}
\hline & $\begin{array}{l}\text { Ciprofloxacin } \\
(n=41)\end{array}$ & $\begin{array}{l}\text { Imipenem } \\
(n=34)\end{array}$ & $p$ value \\
\hline \multicolumn{4}{|l|}{ PPMs } \\
\hline Pseudomonas spp & 14 & 12 & 0.917 \\
\hline Acinetobacter $\mathrm{spp}$ & 6 & 3 & $0.498^{\star}$ \\
\hline Stenotrophomonas maltophilia & 1 & & $1.0^{\star}$ \\
\hline Methicillin resistant Staphylococcus aureus (MRSA) & 1 & & $1.0^{\star}$ \\
\hline \multicolumn{4}{|l|}{ Other Gram negative organisms } \\
\hline Haemophilus influenzae & 8 & 13 & 0.074 \\
\hline Klebsiella pneumoniae & 1 & 1 & $1.0^{\star}$ \\
\hline Enterobacter spp & 3 & & $0.246^{\star}$ \\
\hline Escherichia coli & 1 & 1 & $1.0^{\star}$ \\
\hline Serratia marcescens & 1 & & $1.0^{\star}$ \\
\hline Proteus mirabilis & & 1 & $1.0^{\star}$ \\
\hline Citrobacter freundii & 1 & & $1.0^{\star}$ \\
\hline \multicolumn{4}{|l|}{ Gram positive organisms } \\
\hline Streptococcus pneumoniae & 7 & 8 & 0.486 \\
\hline Methicillin sensitive Staphyloccus aureus (MSSA) & 1 & & $1.0^{\star}$ \\
\hline \multicolumn{4}{|l|}{ Fungi } \\
\hline Aspergillus fumigatus & 1 & & $1.0^{\star}$ \\
\hline \multicolumn{4}{|l|}{ Non-PPMs } \\
\hline Coagulase negative Staphylococcus spp & 5 & 7 & 0.326 \\
\hline Streptococcus spp & & 1 & $1.0^{\star}$ \\
\hline Enterococcus faecalis & 2 & 4 & $0.401^{\star}$ \\
\hline Corynebacterium spp & & 1 & $1.0^{\star}$ \\
\hline Achromobacter spp & & 1 & $1.0^{\star}$ \\
\hline Neisseria spp & & 1 & $1.0^{\star}$ \\
\hline Other non-PPMs & 1 & & $1.0^{\star}$ \\
\hline \multicolumn{4}{|l|}{ Fungi } \\
\hline Candida albicans & 1 & & $1.0^{\star}$ \\
\hline
\end{tabular}

All had at least one potentially pathogenic microorganism (PPM) with growth above the defined thresholds in quantitative bacterial cultures (see definition of study population).

Total number of microorganisms recovered does not equal the number of patients in every group due to polybacterial growth.

$\mathrm{p}$ values were calculated with $\chi^{2}$ test or Fisher's exact test ${ }^{\star}$ where appropriate.

growth was similar in both study arms (ciprofloxacin: 10/41 (24\%), imipenem: 8/34 $(24 \%) ; \mathrm{p}=\mathrm{NS})$.

Analysis of clinical response

Fifty six of the 75 patients (75\%) showed a successful clinical response and the clinical response rate with ciprofloxacin $(71 \%)$ was not statistically different from that with imipenem (79\%, $95 \%$ CI -10.8 to 28.1 , table 3$)$. P aeruginosa was the causative agent in 26 of the 75 cases $(35 \%)$ and the clinical response with ciprofloxacin $(10 / 14,71 \%)$ was not significantly different from that with imipenem $(8 / 12$, $67 \%, \mathrm{p}=1.0)$ in this subgroup of patients (table 3). Enterobacteriaceae were causative agents in seven cases $(9 \%)$ and the success rate did not differ significantly between treatment arms (table 3).

\section{Analysis of bacteriological response}

The bacteriological response was rated a success in 37 patients (49\%) and was not significantly different between the two treatment groups (table 3, ciprofloxacin 20/41 (49\%), imipenem 17/34 (50\%)). Analysis of the treatment failures showed persistence of the microorganism more often in the imipenem group $(8 / 34(24 \%))$ than in the ciprofloxacin group $(2 / 41(5 \%), 95 \%$ CI 2.9 to $34.4, \mathrm{p}=$ $0.035)$. The frequencies of superinfection $(95 \%$ CI -24.6 to $10.1, \mathrm{p}=0.422)$ and presumed failure $(95 \% \mathrm{CI}-29.7$ to $4.4, \mathrm{p}=$ 0.162 ) were not significantly different between the two study groups (table 3). Analysis of treatment failure due to persistence revealed that eight of 10 patients $(80 \%)$ had $P$ aeruginosa and two (20\%) had Acinetobacter spp in initial bacterial culture. Causative microorganisms for both arms in patients with presumed treatment failure were: $P$ aeruginosa 6/14 (43\%), H influenzae 3/14 (21\%), S pneumoniae, Stenotrophomonas maltophilia, Enterobacter sp, Acinetobacter baumanii, methicillin resistant Staphylococcus aureus, and methicillin sensitive $S$ aureus all 1/14 (7\%).

The bacteriological response rate in the ciprofloxacin arm $(7 / 14,50 \%)$ was not significantly higher than in the imipenem arm (3/12, $25 \%, 95 \%$ CI -60.9 to $10.9, p=0.247)$ in the subgroup of patients with $P$ aeruginosa pneumonia. This was also the case in the subanalysis of cases with Enterobacteriaceae in the baseline culture. Although the bacteriological 
Table 3 Success rates for the clinical and bacteriological responses in the two study arms

\begin{tabular}{|c|c|c|c|}
\hline & Ciprofloxacin & Imipenem & $p$ value ${ }^{\star}(95 \% C I)$ \\
\hline \multicolumn{4}{|l|}{ Clinical response: } \\
\hline Success rate & $29 / 41(71 \%)$ & $27 / 34(79 \%)$ & $0.435(-10.8$ to 28.1$)$ \\
\hline Cure & $17 / 41(42 \%)$ & $22 / 34(65 \%)$ & \\
\hline Improvement & $12 / 41(29 \%)$ & $5 / 34(14 \%)$ & \\
\hline \multicolumn{4}{|c|}{ Pseudomonas aeruginosa $(\mathrm{n}=26)$} \\
\hline Success rate & $10 / 14(71 \%)$ & $8 / 12(67 \%)$ & $1.0(-40.4$ to 30.9$)$ \\
\hline \multicolumn{4}{|l|}{ Enterobacteriaceae $(n=9)$} \\
\hline Success rate & $5 / 6(83 \%)$ & $2 / 3(67 \%)$ & $1.0(-77.8$ to 44.4$)$ \\
\hline \multicolumn{4}{|l|}{ Bacteriological response: } \\
\hline Success & $20 / 41(49 \%)$ & $17 / 34(50 \%)$ & $1.0(-21.5$ to 23.9$)$ \\
\hline Eradication & $7 / 41(17 \%)$ & $10 / 34(29 \%)$ & \\
\hline Presumed eradication & $13 / 41(32 \%)$ & $7 / 34(21 \%)$ & \\
\hline Failure & $21 / 41(51 \%)$ & $17 / 34(50 \%)$ & \\
\hline Persistence & $2 / 41(5 \%)$ & $8 / 34(24 \%)$ & \\
\hline Superinfection & $9 / 41(22 \%)$ & $5 / 34(15 \%)$ & \\
\hline Presumed failure & $10 / 41(24 \%)$ & $4 / 34(11 \%)$ & \\
\hline \multicolumn{4}{|c|}{ Pseudomonas aeruginosa $(\mathrm{n}=26)$} \\
\hline \multirow{2}{*}{\multicolumn{4}{|c|}{ Enterobacteriaceae $(n=9)$}} \\
\hline & & & \\
\hline Success rate & $5 / 6(83 \%)$ & $1 / 3(33 \%)$ & $0.266(-111.1$ to 11.1$)$ \\
\hline
\end{tabular}

success rate was $83 \%$ in the ciprofloxacin group (5/6 cases) compared with $33 \%(1 / 3$ cases) in the imipenem group, the number of observations was too small to yield a significant difference ( $p=0.266,95 \%$ CI -111.1 to 11.1 ).

\section{Development of resistance}

Development of resistance was observed in five of 26 patients (19\%) with initial isolates of $P$ aeruginosa with no significant differences between treatment groups (ciprofloxacin 1/14 $(7 \%)$ versus imipenem $4 / 12(33 \%) ; \mathrm{p}=0.147)$. Acinetobacter spp were causative microorganisms in nine patients but only three (33\%) had evaluable follow up cultures. In these three patients no resistance developed during the study period. No resistant strains of Enterobacteriaceae were isolated in eight evaluable follow up cultures.

\section{Adverse effects and mortality}

A total of 10 adverse events were reported in 75 patients (13\%) during the study period which were rated to have a possible relationship with the study drug. These figures did not differ significantly between the treatment groups (ciprofloxacin 6/41 (15\%), imipenem 4/34 (12\%); p $=0.494)$. The adverse events reported in the ciprofloxacin group were septic shock $(n=3)$, gastrointestinal bleeding $(\mathrm{n}=1)$, renal failure $(\mathrm{n}=1)$, and angina $(\mathrm{n}=1)$. In the imipenem group cardiac arrest $(n=1)$, a significant increase in laboratory variables $(n=1)$, and multiorgan failure $(n=1)$ were reported. No seizures were observed during the study in either treatment group. The overall crude mortality was $12 / 75(16 \%)$ with no significant differences between treatment arms (ciprofloxacin 8/41 (20\%), imipenem 4/34 (12\%); p $=0.362)$. The most frequent causes of death were shock $(8 / 29,18 \%)$, multiple organ failure $(5 / 29,17 \%)$, cardiac arrest $(4 / 29,14 \%)$, and respiratory failure $(2 / 29,7 \%)$ with no significant differences between the treatment groups.
ANALYSIS OF THE INTENT-TO-TREAT POPULATION The mean duration of treatment was not significantly different between the treatment groups in the intent-to-treat population (ciprofloxacin 8.4 (4.2) days versus imipenem 9.3 (4.2) days; $p=0.297)$. The clinical response was evaluable in 109 of the 149 patients $(73 \%)$ (ciprofloxacin 52/72 (72\%), imipenem 57/77 $(74 \%) ; p=0.803)$ and seven of the 149 patients $(5 \%)$ had no evaluable basal bacterial culture (ciprofloxacin 3/72 (4\%), imipenem $4 / 77(5 \%) ; \mathrm{p}=0.755)$. At least one PPM was recovered in 48 of 69 patients in the ciprofloxacin group $(70 \%)$ and in 43 of 73 patients in the imipenem group $(59 \% ; \mathrm{p}=0.780)$. The predominantly recovered causative organisms in the study population were $P$ aeruginosa $(\mathrm{n}=$ 32), H influenzae ( $\mathrm{n}=24)$, and $S$ pneumoniae (n $=15)$.

The overall clinical success rate in the intent-to-treat population (74/109 evaluable patients, 68\%) was not significantly different from the success rate in the study population $(75 \%, 95 \% \mathrm{CI}-6.1$ to $20.1, \mathrm{p}=0.321)$. The clinical success rate of treatment with imipenem (40/57, 70\%) was not significantly higher than the success rate with ciprofloxacin $(34 / 52,65 \%, 95 \%$ CI -12.8 to $22.3 ; \mathrm{p}=$ $0.746)$.

A total of 35 adverse events were reported with no significant difference between the study arms (ciprofloxacin 21/72 (29\%), imipenem $14 / 77(18 \%) ; p=0.113)$. Changes in laboratory values of possible clinical significance were 5/21 (24\%) reported adverse effects in the ciprofloxacin group and 6/14 (42\%) in the imipenem group $(\mathrm{p}=0.283)$. Shock and multiorgan failure were reported in 5/14 (36\%) events in the ciprofloxacin group and $3 / 14$ $(42 \%)$ events in the imipenem group $(p=$ $0.577)$.

\section{Discussion}

The success rate of the clinical response to treatment of severe nosocomial pneumonia in patients requiring mechanical ventilation was not significantly different between ciprofloxacin (29/41, 71\%) and imipenem (27/34, $79 \%)$. This was true for the study population and the intent-to-treat population. No differences were found in the bacterial response rate to ciprofloxacin $(20 / 49,49 \%)$ or imipenem $(17 / 34,50 \%)$ in this study population.

Despite the introduction of potent broad spectrum antimicrobial agents and the use of preventive measures, nosocomial pneumonia remains an important cause of mortality and morbidity in the ICU. ${ }^{28} 29$ The causative microorganism varies according to the individual patient risk profile. The severity, type, and number of risk factors and the time of onset of nosocomial pneumonia may influence the risk profiles. Gram negative bacilli, Enterobacteriaceae, H influenzae, and methicillin sensitive $S$ aureus are frequent causative agents in nosocomial pneumonia. P aeruginosa and other potentially drug resistant microorganisms play an important part in patients receiving prolonged mechanical ventilation and antibiotic pretreatment. ${ }^{30}$ Nosocomial pneumonia is sen- 
sitive to empirical treatment with parenteral broad spectrum antibiotic drugs, but the use of a single drug is controversial. ${ }^{1}$ Single drug treatment may be less efficient in patients with $P$ aeruginosa or polymicrobial growth, but it may also be beneficial by reducing toxicity and expense. $^{31}$

The clinical success rate for both drugs was good in our study population, with no differences between the two. Our study included a very homogeneous study population consisting only of patients with microbiologically confirmed nosocomial pneumonia on mechanical ventilation. This is an important difference from previous studies as we used lower respiratory tract specimens together with quantitative bacterial cultures to confirm pneumonia. We could not confirm the superiority of ciprofloxacin over imipenem as described by Fink et al in a large population of patients with a clinical diagnosis of community or nosocomial pneumonia on and off mechanical ventilation. ${ }^{24}$ On the contrary, in our patients with severe nosocomial pneumonia there was a trend in favour of imipenem but our study was too small to detect small differences. However, the percentage of positive clinical responses in our study was comparable to theirs ${ }^{24}$ and to other studies investigating treatment with a single antibiotic of the same class in at least in one arm. Cometta et $a l^{12}$ compared the efficacy of imipenem with a combination of imipenem plus netilmicin in patients with severe nosocomial pneumonia or sepsis and reported a clinical success rate of $80 \%$ with imipenem alone and $86 \%$ with the combination. Colardyn and Faulkner compared two carbapenem regimens in the treatment of nosocomial bacterial infection. ${ }^{11}$ The clinical response rates were comparable for imipenem/ cilastatin (75\%) and meropenem (75\%) in the subanalysis of nosocomial pneumonia.

As in other studies, the bacteriological eradication rates were substantially lower than the clinical success rates but were also comparable for both treatments. Although the clinical success rates were generally not affected by the recovery of Gram negative bacilli, certain restrictions with respect to the bacteriological response apply. When $P$ aeruginosa was identified as the causative agent in the baseline culture there was a non-significant trend towards a lower eradication rate with imipenem $(25 \%$ versus $50 \% ; \mathrm{p}=0.247)$. This observation is in accordance with the results of Fink et al who found a better eradication rate with ciprofloxacin than with imipenem in the presence of $P$ aeruginosa in a multivariate model. ${ }^{24}$ However, we used extremely stringent entry criteria and the number of cases was too small in the subanalysis of patients with $P$ aeruginosa to confirm conclusively the results of Fink et al. ${ }^{24}$

The development of resistance is a major concern when single antibiotics are prescribed. $P$ aeruginosa resistance developed in five of 26 patients $(19 \%)$ with no significant differences between the treatment arms. However, even combination therapy with an antipseudomonal agent may not result in bacteriological cure. Cometta and coworkers found the emergence of $P$ aeruginosa resistant to the study drug to be less frequent with imipenem alone $(9 \%)$ than with imipenem plus netilmicin $(15 \%) .{ }^{12}$ No case of resistance of Enterobacteriaceae was identified in our study. However, the number of evaluable cultures with Enterobacteriaceae was small and the data must be interpreted with caution.

Both ciprofloxacin and imipenem in the doses administered could be regarded as safe and the incidence of severe adverse events was not significantly different between the two drugs. The absence of seizures even with high doses in this study may be due to sedation during mechanical ventilation. The overall mortality was $16 \%$ which did not differ between the two arms of the study.

Some possible limitations of this study should be considered. Firstly, our study was considerably smaller than the investigation published by Fink et $a l^{24}$ and does not therefore have the power to detect small differences. However, our study analysed only patients with microbiologically confirmed pneumonia, which has become the diagnostic standard. Secondly, the lower respiratory tract of all patients was sampled before starting the study medication. We were therefore able to adjust the antibiotic in cases with resistant microorganisms. The number of patients with resistant microorganisms was small, but we have to assume that these cases would have been undetected without microbiological examination. Treatment with a single antibiotic may therefore not be the ideal empirical choice for patients with severe nosocomial pneumonia in institutions without routine microbiological sampling. Thirdly, we could not establish differences between the treatment groups in a subanalysis of patients with $P$ aeruginosa in the baseline culture. However, this was not the primary objective of the study and larger trials are needed to study this further.

In conclusion, treatment with either ciprofloxacin or imipenem yielded good clinical success rates in selected patients with microbiologically confirmed nosocomial pneumonia on mechanical ventilation. We were not able to reach conclusive results from the subanalysis of patients with nosocomial pneumonia caused by $P$ aeruginosa. Physicians should be prepared to broaden antibiotic regimens in these cases because insufficient data are available to rule out the possibility that treatment with a single antibiotic facilitates the development of resistance in patients with nosocomial pneumonia caused by $P$ aeruginosa.

This study was supported by Bayer Spain, Fundació Clínic/ CIRIT, and IDIBAPS, Barcelona, Spain, the European Respiratory Society (ERS), and the Bochumer Arbeitskreis für Pneumologie und Allergologie (BAPA). The trial registry number was BAY Q 3939/0497.

TB was supported during 1999 by an educational grant from IDIBAPS, Barcelona, Spain and the European Respiratory Society (ERS) and during 2000 by the Bochumer Arbeitskreis für Pneumologie und Allergologie (BAPA).

1 American Thoracic Society. Hospital-acquired pneumonia in adults: diagnosis, assessment, initial therapy, and prevention. A consensus statement. Am $\mathcal{F}$ Respir Crit Care prevention. A consensus

2 Alvarez-Lerma F, ICU-acquired Pneumonia Study Group. Modification of empiric antibiotic treatment in patients 
with pneumonia acquired in the intensive care unit. Intensive Care Med 1996;22:387-94.

Gudiol F, Cabellos C, Pallares $\mathrm{R}$, et al. Intravenous ciprofloxacin therapy in severe infections. Am F Med 1989; 87:221-4S

4 Peloquin CA, Cumbo TJ, Nix DE, et al. Intravenous ciprofloxacin in patients with nosocomial lower respiratory tract infections: Impact of plasma concentrations, organism MIC, and clinical condition on bacterial eradication. Arch Intern Med 1989;149:2269-73.

5 Potgieter PD, Linton DM, Forder AA, et al. Imipenem/ cilastatin in the treatment of severe nosocomial pneumonia. S Afr Med ₹ 1988;74:390-2.

6 Salata RA, Gebhart RL, Palmer DL, et al. Pneumonia treated with imipenem. Am f Med 1985;78:104-9.

7 Unertl K, Ruckdeschel G, Forst $\mathrm{H}$, et al. Imipenem cilastatin for the treatment of respiratory tract infections in patients with ventilatory support. Infection 1986;14:176patie 80 .

8 Beasley CR, Humble MW, O'Donnell TV. Treatment of pneumonia with imipenem/cilastatin. NZ Med 7 1985;98: 494-7.

9 Rapp RP, Young B, Bertch K, et al. Clinical outcome of nosocomial pneumonia following imipenem/cilastatin therapy. Drug Intell Clin Pharm 1987;21:272-6.

10 Ernst JA, Sy ER, Colon-Lucca H, et al. Ciprofloxacin in the treatment of pneumonia. Antimicrob Agents Chemother 1986;29:1088-9.

11 Colardyn F, Faulkner KL, Meropenem Serious Infection Study Group. Intravenous meropenem versus imipenem cilastatin in the treatment of serious bacterial infections in hospitalized patients. F Antimicrob Chemother 1996;38:52337.

12 Cometta A, Baumgartner JD, Lew D. Prospective randomized comparison of imipenem monotherapy with imipenem plus netilmycin for treatment of severe infections in nonneutropenic patients. Antimicrob Agents Chemother 1994;38:1309-13.

13 Hartenauer U, Weilemann LS, Bodmann KF, et al. Comparative clinical trial of ceftazidime and imipenem/ Comparative clinical trial of ceftazidime and imipenem/ and septicaemias. F Hosp Infect 1990;15:61-4.

14 Khan FA, Basir R. Sequential intravenous-oral administration of ciprofloxacin vs ceftazidime in serious bacteria respiratory tract infections. Chest 1989;96:528-37.

15 Rapp RP, Bileter $M$, Hatton J, et al. Intravenous ciprofloxacin versus ceftazidime for the treatment of nosocomial pneumonia and urinary tract infections. Clin Pharm 1991, 10:49-55.

16 Trenholme GM, Schmitt BA, Spear J, et al. Randomized study of intravenous/oral ciprofloxacin versus ceftazidime in the treatment of hospital and nursing home patients with lower respiratory tract infections. Am $\mathcal{F}$ Med 1989;87:116$8 \mathrm{~S}$
17 Diaz-Mitoma F, Harding GKM, Louie TJ, et al. Prospective randomized comparison of imipenem/cilastatin and cefo(axime for treatment of lung, soft tis

18 Baumgartner JD, Glauser MP. Comparative study of imipenem in severe infections. F Antimicrob Chemother 1983;12:141-8

19 Stamboulian D, Arguello EA, Jasovich A, et al. Comparative clinical evaluation of imipenem/cilastatin vs. cefotaxime in the treatment of severe bacterial infections. Rev Infect Dis 1985; 7:S458-62.

20 Haddow A, Greene S, Heinz G, et al. Ciprofloxacin (intravenous/oral) versus ceftazidime in lower respiratory tract infections. Am f Med 1989;87:113-5S

21 Menon L, Ernst JA, Sy ER, et al. Sequential intravenous/oral ciprofloxacin compared with intravenous ceftazidime in the treatment of serious lower respiratory tract infections. $A m \mathcal{F}$ Med 1989;87:119-20S

22 Lode H, Wiley R, Hoffken G, et al. Prospective randomized controlled study of ciprofloxacin versus imipenemcilastatin in severe clinical infections. Antimicrob Agents Chemother 1987;31:1491-6.

23 Polk HCJ, Livingston DH, Fry DE, et al. Treatment of pneumonia in mechanically ventilated trauma patients. Results of a prospective trial. Arch Surg 1997,132:1086-92. 24 Fink MP, Snydman DR, Niederman MS, et al. Treatment of severe pneumonia in hospitalized patients: results of a multicenter, randomized, double blind trial comparing intravenous ciprofloxacin with imipenem/cilastatin. F Antimicrob Chemother 1994;38:547-57.

25 Meduri GU, Chastre J. The standardization of bronchocopic techniques for ventilator-associated pneumonia. Chest 1992;102:557-64S.

26 El-Ebiary M, Torres A, González J, et al. Quantitative cultures of endotracheal aspirates for the diagnosis of ventilator-associated pneumonia. Am $\mathcal{f}$ Respir Crit Care Med 1993;147:1552-7.

27 Performance standards for antimicrobial susceptibility testing: 8th informational supplement. NCCLS document M100-SB. informational supplement. NCCLS document M100-SB. Standards, 1998.

28 Craven DE, Steger KA, Barber TW. Preventing nosocomial pneumonia: state of the art and perspectives for the 1990s. Am 7 Med 1991;91(Suppl 3B):44-53S.

29 Torres A, Aznar R, Gatell JM, et al. Incidence, risk, and prognosis factors of nosocomial pneumonia in mechanically ventilated patients. Am Rev Respir Dis 1990;142:5238.

30 Trouillet JL, Chastre J, Vuagnat A, et al. Ventilatorassociated pneumonia caused by potentially drug-resistan bacteria. Am F Respir Crit Care Med 1998;157:531-9.

31 Arbo MDJ, Snydman DR. Monotherapy is appropriate for nosocomial pneumonia in the intensive care unit. Semin Respir Infect 1993;8:259-67. 